\title{
HOMOTOPY PERTURBATION METHOD COMBINED WITH ZZ TRANSFORM TO SOLVE SOME NONLINEAR FRACTIONAL DIFFERENTIAL EQUATIONS
}

\section{LAKHDAR RIABI* KACEM BELGHABA, MOUNTASSIR HAMDI CHERIF, DJELLOUL ZIANE}

Laboratory of Mathematics and Its Applications (LAMAP), Université Oran1, Oran 31000, Algeria

${ }^{*}$ Corresponding author: mountassir27@yahoo.fr

\begin{abstract}
The idea proposed in this work is to extend the ZZ transform method to resolve the nonlinear fractional partial differential equations by combining them with the so-called homotopy perturbation method (HPM). We apply this technique to solve some nonlinear fractional equations as: nonlinear time-fractional Fokker-Planck equation, the cubic nonlinear time-fractional Schrödinger equation and the nonlinear timefractional $\mathrm{KdV}$ equation. The fractional derivative is described in the Caputo sense. The results show that this is the appropriate method to solve somme models of nonlinear partial differential equations with time-fractional derivative.
\end{abstract}

\section{INTRODUCTION}

Fractional calculus is a field of applied mathematics that deals with derivatives and integrals of arbitrary orders. During the last decade, fractional calculus has found applications in numerous seemingly diverse fields of science and engineering. Fractional differential equations are increasingly used to model problems in fluid mechanics, acoustics, biology, electromagnetism, diffusion, signal processing, and many other physical processes [14].

Since the physical side is often associated with fractional differential equations as explained in the previous paragraph, many researchers have used existing methods to solve this type of equations, others are trying

Received 2019-01-30; accepted 2019-03-18; published 2019-05-01.

2010 Mathematics Subject Classification. 26A33, 44A05, 34K37, 35F61.

Key words and phrases. Caputo fractional derivative; homotopy perturbation method; ZZ transform; Fokker-Plank Equation; Schrödinger equation; KdV equation.

(C)2019 Authors retain the copyrights of their papers, and all open access articles are distributed under the terms of the Creative Commons Attribution License. 
to discover new methods faster than existing methods, and all this to facilitate the resolution of this type of equations, especially non-linear ones. These efforts have strengthened this area of research through many methods, among them we find homotopy perturbation method (HPM). This method was established in 1998 by J.H. He ( [10], [11], [12]) and applied to various linear and nonlinear differential equations, its application was also extended to linear and nonlinear fractional differential equations.

Then, a new option emerged recently, includes the composition of Laplace transform, Sumudu transform, Natural Transform or Elzaki transform with this method for solving linear and nonlinear differential equations. Among which are the homotopy perturbation transform method [13], homotopy perturbation Sumudu transform method [17], Natural homotopy perturbation method [1], homotopy perturbation Elzaki transform $\operatorname{method}([3],[8])$.

The objective of the present study is to combine two powerful methods, homotopy perturbation method and ZZ transform method to get a better method to solve nonlinear fractional partial differential equations. The modified method is called fractional homotopy perturbation ZZ transform method (FHPZZTM) and we will apply them to solve the following nonlinear time-fractional Fokker-Planck equation, the cubic nonlinear time-fractional Schrödinger equation and the time-fractional KdV equation.

The present paper has been organized as follows: In section 2, some basic notions about fractional calculus and basic definitions and properties of the ZZ transform method. In section 3, we give an analysis of the proposed method. In section 4, we present solutions to proposed equations explaining how to apply the proposed method. Finally, the conclusion follows.

\section{BASIC DEFINITIONS}

In this section, we give some basic notions about fractional calculus, ZZ transform and ZZ transform of fractional derivatives which are used further in this paper.

2.1. Fractional calculus. We give some basic definitions and properties of the fractional calculus theory as the Riemann-Liouville fractional integrals and Caputo fractional derivative (see [7], [15]).

Definition 2.1. Let $\Omega=[a, b](-\infty<a<b<+\infty)$ be a finite interval on the real axis $\mathbb{R}$. The RiemannLiouville fractional integral $I_{0+}^{\alpha} f$ of order $\alpha \in \mathbb{R}(\alpha>0)$ is defined by

$$
\begin{aligned}
\left(I_{0+}^{\alpha} f\right)(t) & =\frac{1}{\Gamma(\alpha)} \int_{0}^{t} \frac{f(\tau) d \tau}{(t-\tau)^{1-\alpha}}, \quad t>0, \alpha>0 \\
\left(I_{0+}^{0} f\right)(t) & =f(t) .
\end{aligned}
$$

Here $\Gamma(\cdot)$ is the gamma function. 
Theorem 2.1. Let $\alpha \geqslant 0$ and let $n=[\alpha]+1$. If $f(t) \in A C^{n}[a, b]$, then the Caputo fractional derivative $\left({ }^{c} D_{0+}^{\alpha} f\right)(t)$ exist almost everywhere on $[a, b]$. If $\alpha \notin \mathbb{N},\left({ }^{c} D_{0^{+}}^{\alpha} f\right)(t)$ is represented by

$$
\left({ }^{c} D_{0+}^{\alpha} f\right)(t)=\frac{1}{\Gamma(n-\alpha)} \int_{0}^{t} \frac{f^{(n)}(\tau) d \tau}{(t-\tau)^{\alpha-n+1}}
$$

where $D=\frac{d}{d x}$ and $n=[\alpha]+1$.

Remark 2.1. In this paper, we consider the time-fractional derivative in the Caputo's sense. When $\alpha \in \mathbb{R}^{+}$, the time-fractional derivative is defined as

$$
\begin{aligned}
\left({ }^{c} D_{t}^{\alpha} u\right)(x, t) & =\frac{\partial^{\alpha} u(x, t)}{\partial t^{\alpha}} \\
& =\left\{\begin{aligned}
& \frac{1}{\Gamma(m-\alpha)} \int_{0}^{t}(t-\tau)^{m-\alpha-1} \frac{\partial^{m} u(x, \tau)}{\partial \tau^{m}}, m-1<\alpha<m, \\
& \frac{\partial^{m} u(x, t)}{\partial t^{m}}, \quad \alpha=m,
\end{aligned}\right.
\end{aligned}
$$

where $m \in \mathbb{N}^{*}$.

2.2. Definitions and properties of the $\mathbf{Z Z}$ transform. The ZZ transform was defined by Zain Ul Abadin Zafar [20] in 2016. In this section, we give some basic definitions and properties of this transform (see [20]).

Definition 2.2. Let $u(t)$ be a function defined for all $t \geq 0$. The $Z Z$ transform of $u(t)$ is the function $T(v, s)$ defined by

$$
Z[u(t)]=T(v, s)=s \int_{0}^{\infty} u(v t) e^{-s t} d t
$$

Theorem 2.2. If $u(t)$ is piecewise continuous in every finite interval $0 \leq t \leq K$ and of exponential order $\gamma$ for $t>K$, then its $Z Z$ transform $T(v, s)$ exists for all $s>\gamma, v>\gamma$.

Proof. (see [20])

2.2.1. Some properties of the $Z Z$ transform

. 1. The ZZ transform of the $n^{t h}$ derivative of $u(t)$ is given by

$$
Z\left[u^{(n)}(t)\right]=\frac{s^{n}}{v^{n}} Z[u(t)]-\sum_{k=0}^{n-1} \frac{s^{n-k}}{v^{n-k}} u^{(k)}(0) .
$$

2. Some elementary functions and their transformations 


\begin{tabular}{|l|l|}
\hline$u(t)$ & $Z[u(t)]$ \\
\hline 1 & 1 \\
\hline$t$ & $\frac{v}{s}$ \\
\hline$t^{n}$ & $n ! \frac{v^{n}}{s^{n}}, n=0,1,2, \ldots$ \\
\hline$t^{\alpha}$ & $\Gamma(\alpha+1) \frac{v^{\alpha}}{s^{\alpha}}, \alpha \geqslant 0$. \\
\hline
\end{tabular}

Theorem 2.3. The ZZ transform of the time-fractional derivative in the Caputo's sense is defined as

$$
Z\left[\left({ }^{c} D_{0+}^{\alpha} u\right)(t) ;(v, s)\right]=\frac{s^{\alpha}}{v^{\alpha}} Z[u(t)]-\sum_{k=0}^{n-1} \frac{s^{\alpha-k}}{v^{\alpha-k}} u^{(k)}(0) \quad, \quad n-1<\alpha \leq n, \quad n=1,2, \ldots
$$

Proof. (see [19])

\section{Fractional homotopy perturbation ZZ transform method}

To illustrate the basic idea of this method, we consider a general nonlinear fractional partial differential non homogeneous equation with initial conditions of the form

$$
{ }^{c} D_{t}^{\alpha} u(x, t)+R u(x, t)+N u(x, t)=g(x, t),
$$

where $t>0, x \in \mathbb{R}, 0<\alpha \leq 1$ and the initial conditions

$$
u(x, 0)=h(x)
$$

${ }^{c} D_{t}^{\alpha} u(x, t)$, is the Caputo fractional derivative of the function $u(x, t), R$ is the linear differential operator, $N$ represent the general nonlinear differential operator, and $h(x, t)$ is the source terms.

Applying the ZZ transform on both sides of (3.1) and using the differentiation property of this transform (2.4), we obtain

$$
Z[u(x, t)]=h(x)+\frac{v^{\alpha}}{s^{\alpha}} Z[g(x, t)]-\frac{v^{\alpha}}{s^{\alpha}} Z[R u(x, t)+N u(x, t)] .
$$

Taking the inverse ZZ transform on both sides of equations in system (3.3) and then by using initial conditions (3.2), we have

$$
u(x, t)=G(x, t)-Z^{-1}\left(\frac{v^{\alpha}}{s^{\alpha}} Z[R u(x, t)+N u(x, t)]\right),
$$

where $G(x, t)$ represents the terms arising from the nonhomogeneous terms and the prescribed initial conditions. Now, we applying the classical perturbation technique, we can assume that the solution can be expressed as a power series in $p$, as given below 


$$
u(x, t)=\sum_{n=0}^{\infty} p^{n} u_{n}(x, t)
$$

where the homotopy parameter $p$, is considered as a small parameter $(p \in[0,1])$. The nonlinear terms can be decomposed as

$$
N u(x, t)=\sum_{n=0}^{\infty} H_{n}(u)
$$

where $H_{n}$ are He's polynomials [5] of $u_{0}, u_{1}, u_{2}, \ldots, u_{n}$, and they can be calculated by the formulas given below

$$
H_{n}\left(u_{0}, \ldots, u_{n}\right)=\frac{1}{n} \frac{\partial^{n}}{\partial p^{n}}\left[N\left(\sum_{i=0}^{\infty} p^{i} u_{i}\right)\right]_{p=0}, n=0,1,2 \ldots
$$

Using (3.5) and (3.6), we can rewrite (3.4) as

$$
\sum_{n=0}^{\infty} p^{n} u_{n}=G(x, t)-p\left(Z^{-1}\left[\frac{v^{\alpha}}{s^{\alpha}} Z\left[R \sum_{n=0}^{\infty} p^{n} u_{n}+\sum_{n=0}^{\infty} p^{n} H_{n}(u)\right]\right]\right) .
$$

This is a coupling of the ZZ transform and homotopy perturbation methods (ZZHPM) using He's polynomials. Now, equating the coefficient of corresponding power of $p$ on both sides of (3.8), we get

$$
\begin{gathered}
u_{0}(x, t)=G(x, t), \\
\vdots \\
u_{n}(x, t)=-Z^{-1}\left(\frac{v^{\alpha}}{s^{\alpha}} Z\left[R u_{n-1}(x, t)+H_{n-1}(u)\right]\right),
\end{gathered}
$$

where $n \in \mathbb{N}^{*}$. We continue in this manner to obtain the general recursive relations.

Finally, the approximate solution is calculated by

$$
u(x, t)=\lim _{N \rightarrow \infty} \sum_{n=0}^{N} u_{n}(x, t) .
$$

The convergence of the series (3.10) has been proved in ( [4], [5]).

\section{Applications of Fractional homotopy PERTURBATion ZZ TRANSFORM METhOD}

In this section, we apply fractional homotopy perturbation ZZ transform method with the Caputo fractional derivative to solve nonlinear time-fractional Fokker-Planck equation, nonlinear time-fractional Schrödinger equation and nonlinear time-fractional KdV equation.

Example 4.1. We consider the following nonlinear time-fractional Fokker-Planck equation

$$
{ }^{c} D_{t}^{\alpha} u=\left(\frac{x}{3} u\right)_{x}-\left(\frac{4}{x} u^{2}\right)_{x}+\left(u^{2}\right)_{x x}, 0<\alpha \leq 1,
$$


with the initial condition

$$
u(x, 0)=x^{2}
$$

By applying ZZ transform of (4.1), we obtain

$$
Z\left[D_{t}^{\alpha} u\right]=Z\left[\left(\frac{x}{3} u\right)_{x}\right]-Z\left[\left(\frac{4}{x} u^{2}\right)_{x}\right]+Z\left[\left(u^{2}\right)_{x x}\right]
$$

Using the differentiation property of the ZZ transform in (4.1), we get

$$
Z[u(x, t)]=x^{2}+\frac{v^{\alpha}}{s^{\alpha}}\left(Z\left[\left(\frac{x}{3} u\right)_{x}\right]-Z\left[\left(\frac{4}{x} u^{2}\right)_{x}\right]+Z\left[\left(u^{2}\right)_{x x}\right]\right) .
$$

Taking the inverse ZZ transform on both sides of (4.4), we obtain

$$
u(x, t)=x^{2}+Z^{-1}\left[\frac{v^{\alpha}}{s^{\alpha}}\left(Z\left[\left(\frac{x}{3} u\right)_{x}\right]-Z\left[\left(\frac{4}{x} u^{2}\right)_{x}\right]+Z\left[\left(u^{2}\right)_{x x}\right]\right)\right]
$$

By applying the aforesaid homotopy perturbation method, we have

$$
\sum_{n=0}^{\infty} p^{n} u_{n}=x^{2}+p\left(Z^{-1}\left[\frac{v^{\alpha}}{s^{\alpha}}\left(Z\left[\left(\frac{x}{3} \sum_{n=0}^{\infty} p^{n} u_{n}\right)_{x}\right]-Z\left[\sum_{n=0}^{p} H_{n}(u)\right]\right)\right]\right) .
$$

Equating the coefficient of the like power of $p$ on both sides in (4.6), we get

$$
\begin{gathered}
p^{0}: u_{0}(x, t)=x^{2} \\
\vdots \\
p^{n}: u_{n}(x, t)=Z^{-1}\left[\frac{v^{\alpha}}{s^{\alpha}}\left(Z\left[\left(\frac{x}{3} \sum_{n=0}^{\infty} p^{n} u_{n}\right)_{x}\right]-Z\left[\sum_{n=0}^{p} H_{n}(u)\right]\right)\right],
\end{gathered}
$$

where $n \in \mathbb{N}^{*}$. The first few components of He's polynomials, are given by

$$
\begin{gathered}
H_{0}=-\left[\frac{4}{x} u_{0}^{2}\right]_{x}+u_{0 x x}^{2}, \\
H_{1}=-\left[\frac{4}{x} 2 u_{0} u_{1}\right]_{x}+2\left(u_{0} u_{1}\right)_{x x}, \\
H_{2}=-\left[\frac{4}{x}\left(2 u_{0} u_{2}+u_{1}^{2}\right)\right]_{x}+2\left(2 u_{0} u_{2}+u_{1}^{2}\right)_{x x}, \\
H_{3}=-\left[\frac{4}{x}\left(2 u_{0} u_{3}+2 u_{1} u_{2}\right)\right]_{x}+2\left(2 u_{0} u_{3}+2 u_{1} u_{2}\right)_{x x}
\end{gathered}
$$

Using He's polynomials (4.8) and the iteration formulas (4.7) we obtain 


$$
\begin{gathered}
u_{0}(x, t)=x^{2} \\
u_{1}(x, t)=x^{2} \frac{t^{\alpha}}{\Gamma(\alpha+1)}, \\
u_{2}(x, t)=x^{2} \frac{t^{2 \alpha}}{\Gamma(2 \alpha+1)} \\
u_{3}(x, t)=x^{2} \frac{t^{3 \alpha}}{\Gamma(3 \alpha+1)} \\
u_{4}(x, t)=x^{2} \frac{t^{4 \alpha}}{\Gamma(4 \alpha+1)},
\end{gathered}
$$

The first four terms of the decomposition series solution for (4.1) are given by

$$
u(x, t)=x^{2}+x^{2} \frac{t^{\alpha}}{\Gamma(\alpha+1)}+x^{2} \frac{t^{2 \alpha}}{\Gamma(2 \alpha+1)}+x^{2} \frac{t^{3 \alpha}}{\Gamma(3 \alpha+1)}+x^{2} \frac{t^{4 \alpha}}{\Gamma(4 \alpha+1)}+\ldots
$$

Hence

$$
u(x, t)=x^{2} \sum_{k=0}^{+\infty} \frac{t^{k \alpha}}{\Gamma(k \alpha+1)}=x^{2} E_{\alpha}\left(t^{\alpha}\right),
$$

where $E_{\alpha}\left(t^{\alpha}\right)$ is a Mittag-leffler function defined as: $E_{\alpha}\left(t^{\alpha}\right)=\sum_{k=0}^{+\infty} \frac{t^{k \alpha}}{\Gamma(k \alpha+1)}$.

At special case, when $\alpha=1$ we obtain

$$
u(x, t)=x^{2} e^{t},
$$

which is the exact solution to the Fokker-Planck equation of (4.1)-(4.2) as presented in [18] by Adomian decomposition method (ADM), in [16] by varational iteration method (VIM) and in [6] by homotopy perturbation method (HPM).

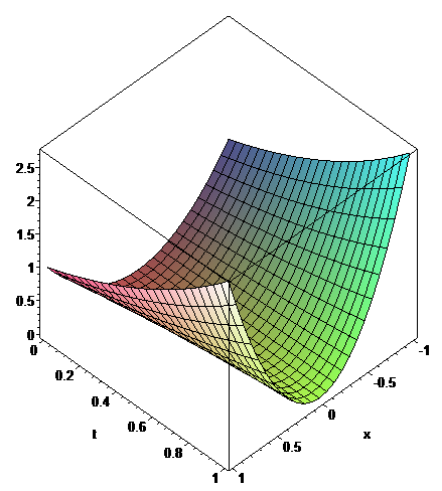

(a)

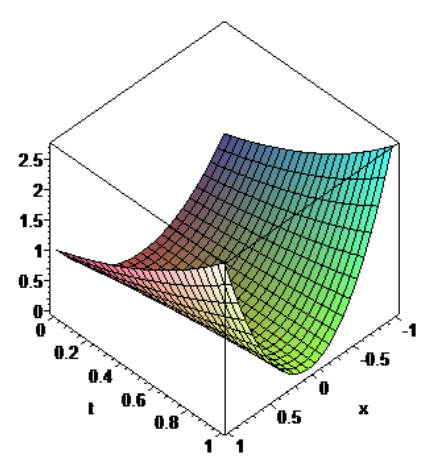

(b)

Figure 1. (a) The exact solution, (b) The approximate solution when $\alpha=1$ of (4.1)-(4.2) 


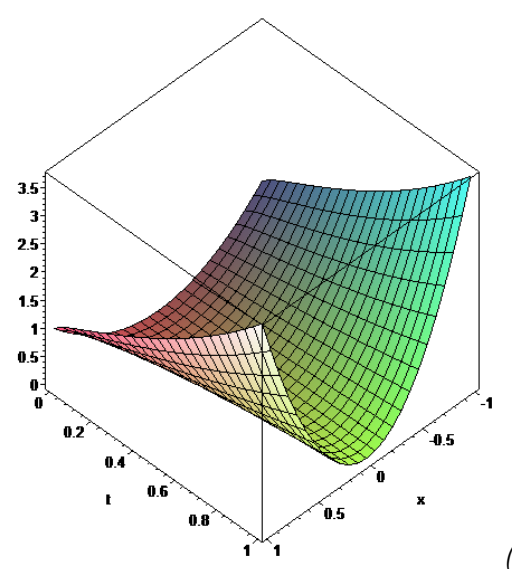

(c)

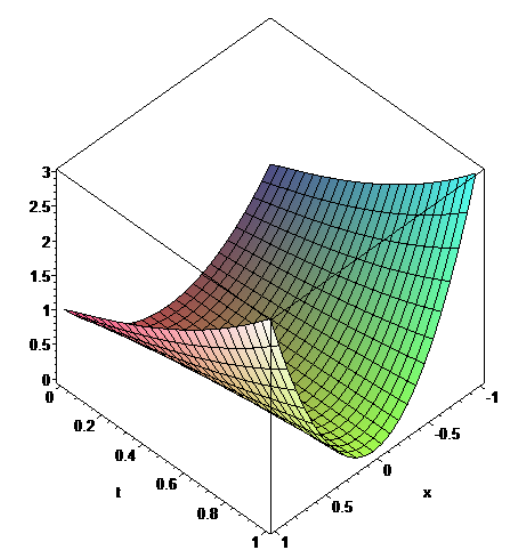

(d)

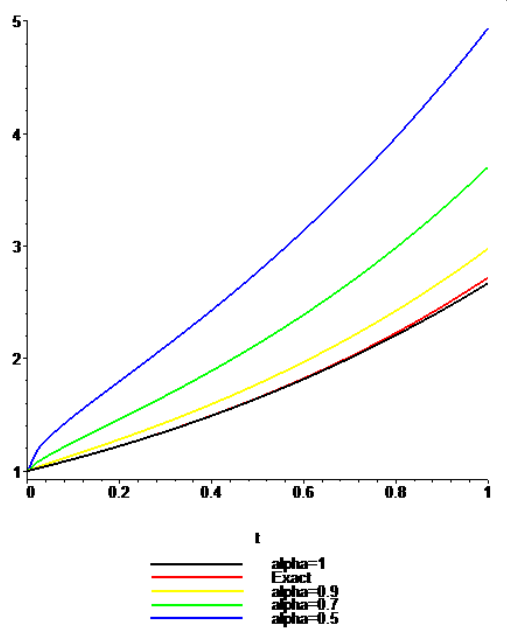

(e)

FigurE 2. (c) The approximate solution when $\alpha=0,7$, (d) The approximate solution when $\alpha=0,9$ of, (e) plots of $u(x, t)$ versus at $x=1$ for different values for $\alpha=1, \alpha=0,9, \alpha=0,7$ and $\alpha=0,5$ of (4.1)-(4.2)

Example 4.2. We consider the following cubic nonlinear time-fractional Schrödinger equation

$$
i^{c} D_{t}^{\alpha} u+u_{x x}-2|u|^{2} u=0,0<\alpha \leq 1
$$

with the initial condition

$$
u(x, 0)=e^{i x}
$$

By applying ZZ transform of (4.11), we obtain

$$
i Z\left[D_{t}^{\alpha} u\right]+Z\left[u_{x x}\right]-2 Z\left[|u|^{2} u\right]=0
$$

Using the differentiation property of the ZZ transform and initial conditions in (4.13), we get

$$
Z[u(x, t)]=e^{i t}+i \frac{v^{\alpha}}{s^{\alpha}}\left(Z\left[u_{x x}\right]-2 i Z\left[|u|^{2} u\right]\right)
$$


Taking the inverse ZZ transform on both sides of (4.14), we obtain

$$
u(x, t)=Z^{-1}\left[\frac{v^{\alpha}}{s^{\alpha}}\left(i Z\left[u_{x x}\right]-2 i Z\left[|u|^{2} u\right]\right)\right] .
$$

By applying the homotopy perturbation method, we have

$$
\sum_{n=0}^{\infty} p^{n} u_{n}=e^{i x}+p\left(Z^{-1}\left[\frac{v^{\alpha}}{s^{\alpha}}\left(i Z\left[\left(\sum_{n=0}^{\infty} p^{n} u_{n}\right)_{x x}\right]-2 i Z\left[\sum_{n=0}^{p} H_{n}(u)\right]\right)\right]\right) .
$$

Equating the coefficient of the like power of $p$ on both sides in (4.16), we get

$$
\begin{gathered}
p^{0}: u_{0}(x, t)=e^{i x} \\
\vdots \\
p^{n}: u_{n}(x, t)=Z^{-1}\left[\frac{v^{\alpha}}{s^{\alpha}}\left(i Z\left[\left(\sum_{n=0}^{\infty} p^{n} u_{n}\right)_{x x}\right]-2 i Z\left[\sum_{n=0}^{p} H_{n}(u)\right]\right)\right],
\end{gathered}
$$

where $n \in \mathbb{N}^{*}$. The nonlinear term is given by

$$
\begin{gathered}
N(u)=|u|^{2} u,|u|^{2}=u \bar{u} \\
N(u)=u^{2} \bar{u} .
\end{gathered}
$$

The first few components of He's polynomials, are given by

$$
\begin{gathered}
H_{0}=u_{0} \overline{u_{0}} \\
H_{1}=2 u_{0} \overline{u_{0}} u_{1}+u_{0}^{2} \overline{u_{1}} \\
H_{2}=2 u_{0} \overline{u_{0}} u_{2}+u_{1}^{2} \overline{u_{0}}+2 u_{0} \overline{u_{1}} u_{1}+u_{0}^{2} \overline{u_{2}}
\end{gathered}
$$

Using He's polynomials (4.19) and the iteration formulas (4.17) we obtain

$$
\begin{gathered}
u_{0}(x, t)=e^{i x} \\
u_{1}(x, t)=-3 i e^{i x} \frac{t^{\alpha}}{\Gamma(\alpha+1)} \\
u_{2}(x, t)=e^{i x} \frac{\left(3 i t^{\alpha}\right)^{2}}{\Gamma(2 \alpha+1)} \\
u_{3}(x, t)=-e^{i x} \frac{\left(3 i t^{\alpha}\right)^{3}}{\Gamma(3 \alpha+1)} \\
u_{4}(x, t)=e^{i x} \frac{\left(3 i t^{\alpha}\right)^{4}}{\Gamma(4 \alpha+1)} \\
\vdots
\end{gathered}
$$

Then the approximate solution in a series form is given by

$$
u(x, t)=e^{i x}-e^{i x} \frac{\left(3 i t^{\alpha}\right)}{\Gamma(\alpha+1)}+e^{i x} \frac{\left(3 i t^{\alpha}\right)^{2}}{\Gamma(2 \alpha+1)}-e^{i x} \frac{\left(3 i t^{\alpha}\right)^{3}}{\Gamma(3 \alpha+1)}+u_{4}(x, t)=e^{i x} \frac{\left(3 i t^{\alpha}\right)^{4}}{\Gamma(4 \alpha+1)}+\ldots
$$

Hence 


$$
u(x, t)=e^{i x} \sum_{k=0}^{+\infty}(-1)^{k} \frac{\left(3 i t^{\alpha}\right)^{k}}{\Gamma(k \alpha+1)}=e^{i x} E_{\alpha}\left(-3 i t^{\alpha}\right) .
$$

At special case, when $\alpha=1$ we obtain

$$
u(x, t)=e^{i(x-3 t)},
$$

which is the exact solution to the cubic nonlinear Schrödinger equation of (4.11) as presented in [2].

Example 4.3. We consider the time-fractional KdV equation

$$
{ }^{c} D_{t}^{\alpha} u-3\left(u^{2}\right)_{x}+u_{x x x}=0,0<\alpha \leq 1
$$

with the initial condition

$$
\begin{gathered}
u(x, 0)=6 x . \\
Z\left[D_{t}^{\alpha} u\right]-3 Z\left[\left(u^{2}\right)_{x}\right]+Z\left[u_{x x x}\right]=0 .
\end{gathered}
$$

Using the differentiation property of the ZZ transform in (4.24), we have

$$
Z[u(x, t)]=6 x+\frac{v^{\alpha}}{s^{\alpha}} Z\left[3\left(u^{2}\right)_{x}-u_{x x x}\right]
$$

Taking the inverse ZZ transform on both sides of (4.25), we obtain

$$
u(x, t)=6 x+Z^{-1}\left[\left[\frac{v^{\alpha}}{s^{\alpha}} Z\left[3\left(u^{2}\right)_{x}-u_{x x x}\right]\right]\right.
$$

By applying the HPM method, we have

$$
\sum_{n=0}^{\infty} p^{n} u_{n}=6 x+p\left(Z^{-1}\left(\frac{v^{\alpha}}{s^{\alpha}} Z\left[3 \sum_{n=0}^{\infty} p^{n}\left(H_{n}(u)\right)_{x}-\sum_{n=0}^{\infty} p^{n} u_{n x x x}\right]\right)\right)
$$

Equating the coefficient of the like power of $p$ on both sides in (4.27), we get

$$
\begin{gathered}
p^{0}: u_{0}(x, t)=6 x \\
\vdots \\
p^{n}: u_{n}(x, t)=Z^{-1}\left(\frac{v^{\alpha}}{s^{\alpha}} Z\left[3\left(H_{n-1}(u)\right)_{x}-\left(u_{n-1}\right)_{x x x}\right]\right),
\end{gathered}
$$

where $n \in \mathbb{N}^{*}$. The first few components of He's polynomials are given by 


$$
\begin{gathered}
H_{0}=u_{0}^{2}, \\
H_{1}=2 u_{0} u_{1}, \\
H_{2}=2 u_{0} u_{2}+u_{1}^{2}, \\
H_{3}=2 u_{0} u_{3}+2 u_{1} u_{2},
\end{gathered}
$$

Using He's polynomials (4.29) and the iteration formulas (4.28) we obtain

$$
\begin{gathered}
u_{0}(x, t)=6 x \\
u_{1}(x, t)=6 x(36) \frac{1}{\Gamma(\alpha+1)} t^{\alpha}, \\
u_{2}(x, t)=6 x(36)^{2} \frac{2}{\Gamma(2 \alpha+1)} t^{2 \alpha} \\
u_{3}(x, t)=6 x(36)^{3} \frac{4 \Gamma^{2}(\alpha+1)+\Gamma(2 \alpha+1)}{\Gamma^{2}(\alpha+1) \Gamma(3 \alpha+1)} t^{3 \alpha} \\
u_{4}(x, t)=6 x(36)^{4}\left(\frac{8 \Gamma^{2}(\alpha+1)+2 \Gamma(2 \alpha+1)}{\Gamma^{2}(\alpha+1) \Gamma(3 \alpha+1)}+\frac{4}{\Gamma(\alpha+1) \Gamma(2 \alpha+1)}\right) \frac{\Gamma(3 \alpha+1)}{\Gamma(4 \alpha+1)} t^{4 \alpha}, \\
\vdots
\end{gathered}
$$

The first four terms of the decomposition series solution for (4.22) is given by

$$
\begin{aligned}
u(x, t)= & 6 x+6 x(36) \frac{1}{\Gamma(\alpha+1)} t^{\alpha}+6 x(36)^{2} \frac{2}{\Gamma(2 \alpha+1)} t^{2 \alpha}+6 x(36)^{3} \frac{4 \Gamma^{2}(\alpha+1)+\Gamma(2 \alpha+1)}{\Gamma^{2}(\alpha+1) \Gamma(3 \alpha+1)} t^{3 \alpha}+ \\
& 6 x(36)^{4}\left(\frac{8 \Gamma^{2}(\alpha+1)+2 \Gamma(2 \alpha+1)}{\Gamma^{2}(\alpha+1) \Gamma(3 \alpha+1)}+\frac{4}{\Gamma(\alpha+1) \Gamma(2 \alpha+1)}\right) \frac{\Gamma(3 \alpha+1)}{\Gamma(4 \alpha+1)} t^{4 \alpha}+\ldots
\end{aligned}
$$

The first four terms of the decomposition series solution, for the special case $\alpha=1$, is given by

$$
u(x, t)=6 x\left[1+(36) t+(36)^{2} t^{2}+(36)^{3} t^{3}+(36)^{4} t^{4}+\ldots\right]
$$

That gives

$$
u(x, t)=\frac{6 x}{1-36 t},|36 t|<1,
$$

which is an exact solution to the KdV equation (4.22)-(4.23) as presented in [21]. 


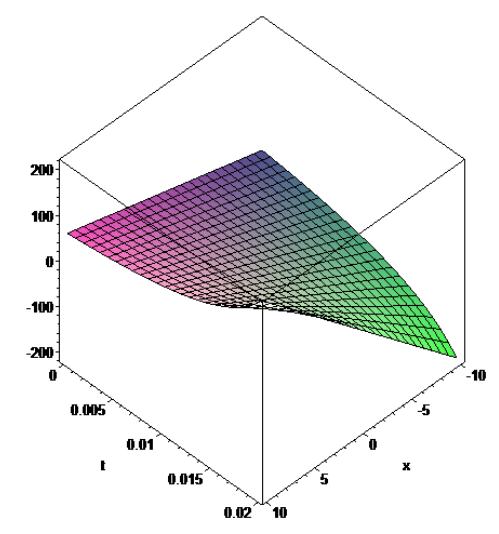

(a)

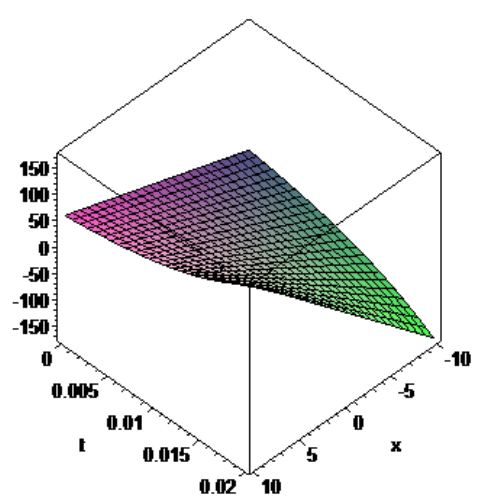

(b)

Figure 3. (a) The exact solution, (b) The approximate solution when $\alpha=1$ of (4.22)-(4.23)

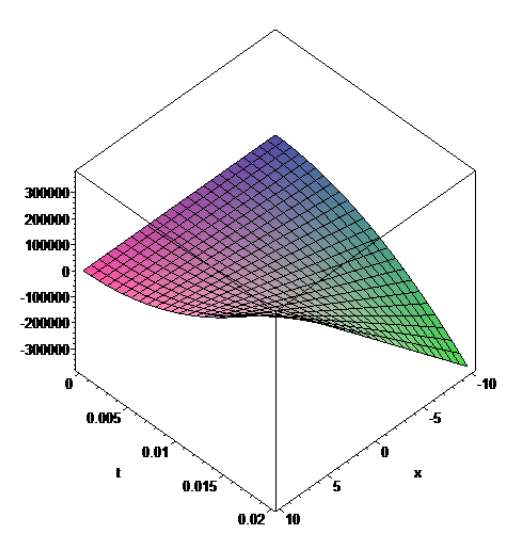

(c)

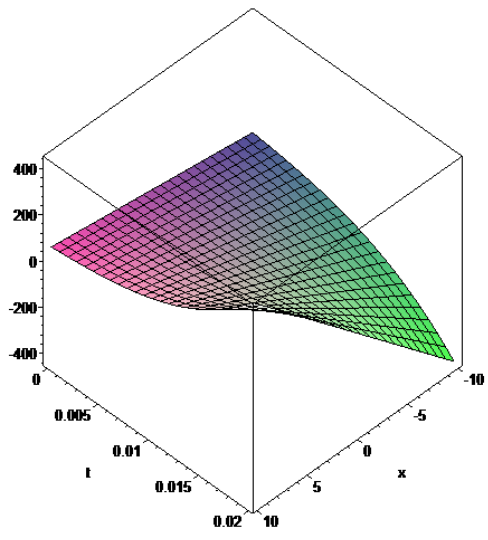

(d)

Figure 4. (c) and (d) The approximate solutions of (4.22)-(4.23) when $\alpha=0.5$ and $\alpha=0.9$ respectively. 


\section{Conclusion}

The coupling of homotopy perturbation method (HPM) and the ZZ transform method, proved very effective to solve nonlinear fractional partial differential equations. The proposed algorithm provides the solution in a series form that converges rapidly to the exact solution if it exists. From the obtained results, it is clear that the HPZZTM yields very accurate solutions using only a few iterates. The results show that the homotopy perturbation ZZ transform method (HPZZTM) can be further implemented to solve other physical models of nonlinear partial differential equations with time-fractional derivative.

\section{REFERENCES}

[1] Adio, A. K., A Reliable Technique for Solving Gas Dynamic Equation using Natural Homotopy Perturbation Method, Glob. J. Sci. Front. Res. (F), 17 (2017), 48-56.

[2] Ayati, Z., Biazar, J., and Ebrahimi, S., A New Homotopy Perturbation Method for Solving Linear and Nonlinear Schrödinger Equations, J. Interpolation Approx. Sci. Comput. 2014 (2014), Art. ID jiasc-00062.

[3] Bhadane, P. K. G., and Pradhan, V. H., Elzaki Transform Homotopy Perturbation Method for Solving Porous Medium Equation, Int. J. Res. Eng. Technol. 2 (2013), 116-119.

[4] Biazar, J., and Aminikhah, H., Study of convergence of homotopy perturbation method for systems of partial differential equations, Comput. Math. Appl. 58 (2009), 2221-2230.

[5] Biazar, J., and Ghazvini, H., Convergence of the homotopy perturbation method for partial differential equations, Nonlinear Anal., Real World Appl. 10 (2009), 2633-2640.

[6] Biazar, J., Hosseini, K., and Gholamin, P., Homotopy Perturbation Method Fokker-Planck Equation. Int. Math. Forum. 19 (2008), 945-954.

[7] Diethelm, K., The Analysis Fractional Differential Equations, Springer-Verlag Berlin Heidelberg, 2010.

[8] Elzaki, T. M., and Hilal, E. M. A., Homotopy Perturbation and Elzaki Transform for Solving Nonlinear Partial Differential Equations, Math. Theory Model. 2 (2012), 33-42.

[9] Ghorbani, A., Beyond Adomian polynomials: He polynomials, Chaos Solitons Fractals, 39 (2009), 1486-1492.

[10] He, J. H., A coupling method of homotopy technique and perturbation technique for nonlinear problems, Int. J. Non-Linear Mech. 35 (2000), 37-43.

[11] He, J. H., Application of homotopy perturbation method to nonlinear wave equations, Chaos Solitons Fractals. 26 (2005), 695-700.

[12] He, J. H., Homotopy perturbation technique, Comput. Meth. Appl. Mech. Eng. 178 (1999), 257-262.

[13] Kumara, S., Yildirim, A., Khan, Y., and Weid, L., A fractional model of the diffusion equation and its analytical solution using Laplace transform, Scientia Iranica B. 19 (2012), 1117-1123.

[14] Mohamed, M. S., Al-Malki, F., and Al-humyani, M., Homotopy Analysis Transform Method for Time-Space Fractional Gas Dynamics Equation, Gen. Math. Notes. 24 (2014), 1-16.

[15] Podlubny, I., Fractional Differential Equations, Academic Press, San Diego, CA, 1999.

[16] Sadhigi, A., Ganji, D., and Sabzehmeidavi, Y., A Study on Fokker-Planck Equation by Variational Iteration Method. Int. J. Nonlinear. Sci. 4 (2007), 92-102.

[17] Singh, J., Kumar, D., and Sushila.,Homotopy Perturbation Sumudu Transform Method for Nonlinear Equations, Adv. Theor. Appl. Mech. 4 (2011), 165-175. 
[18] Tatari, M., Dehghan, M., and Razzaghi, M., Application of Adomain Decomposition Method for the Fokker-Planck Equation. Math. Comp. Model. 45 (2007), 639-650.

[19] Zafar, Z. U. A., Application of ZZ Transform Method on Some Fractional Differential Equations, Int. J. Adv. Eng. Global Technol. 4 (2016), 1355-1363.

[20] Zafar, Z. U. A., ZZ Transform Method, Int. J. Adv. Eng. Glob. Technol. 4 (2016), 1605-1611.

[21] Ziane, D., Belghaba, K., and Hamdi Cherif, M., Fractional Homotopy Perturbation Transform Method for Solving the Time-Fractional KdV,K(2,2) and Burgers Equations, Int. J. Open Probl. Comput. Math. 8 (2015), 63-75. 